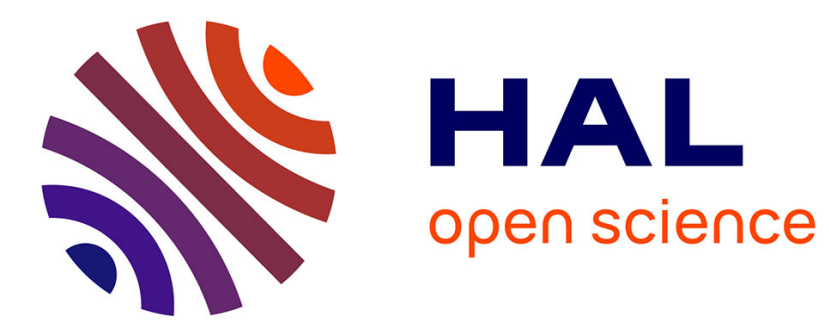

\title{
Influence on the electrolyte resistance of the contact angle of a bubble attached to a disk electrode
} Anh Tuan Tran, François Huet, Kieu Ngo, Philippe Rousseau

\section{To cite this version:}

Anh Tuan Tran, François Huet, Kieu Ngo, Philippe Rousseau. Influence on the electrolyte resistance of the contact angle of a bubble attached to a disk electrode. Journal of electroanalytical chemistry and interfacial electrochemistry, 2015, 737, pp.114 - 122. 10.1016/j.jelechem.2014.07.017 . hal-01111535

\section{HAL Id: hal-01111535 \\ https://hal.science/hal-01111535}

Submitted on 30 Jan 2015

HAL is a multi-disciplinary open access archive for the deposit and dissemination of scientific research documents, whether they are published or not. The documents may come from teaching and research institutions in France or abroad, or from public or private research centers.
L'archive ouverte pluridisciplinaire $\mathbf{H A L}$, est destinée au dépôt et à la diffusion de documents scientifiques de niveau recherche, publiés ou non, émanant des établissements d'enseignement et de recherche français ou étrangers, des laboratoires publics ou privés. 


\title{
Influence on the electrolyte resistance of the contact angle of a bubble attached to a disk electrode
}

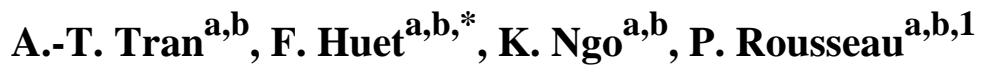 \\ ${ }^{a}$ Sorbonne Universités, UPMC Univ Paris 06, UMR 8235, Laboratoire Interfaces et Systèmes \\ Electrochimiques, F-75005, Paris, France \\ ${ }^{b}$ CNRS, UMR 8235, LISE, F-75005, Paris, France
}

\begin{abstract}
In a previous work, the analysis of the electrolyte-resistance (ER) increment due to the presence of an insulating sphere above or in contact with a disk electrode, simulating a spherical particle, drop, or gas bubble close or attached to an electrode has been reported [Bouazaze et al., Electrochim. Acta 55 (2010) 1645]. In the present work, the influence on the ER of the contact angle with the electrode surface, which plays for example a major role in the size of bubbles on gasevolving electrodes, is quantitatively determined. The mathematical collocation method used in the previous work was improved to account for the change in geometry of the electrode-sphere system. The theoretical results show that the ER increment due to the presence of the sphere depends on its size, position, and contact angle. For a given size and position of spheres of aspect ratio less than 0.4 , the ER increment due to the contact angle varies roughly between $-45 \%$ and $+35 \%$ of the increment due to a perfect sphere, depending on the interplay of the surface and volume competing effects influencing the ER. Despite their low values, the ER increments could be experimentally measured for spheres of different contact angle placed at the electrode centre, owing to a highprecision motorized translation stage and a specific low-noise ER measurement device. An excellent agreement was obtained between the theoretical and experimental results.
\end{abstract}

Keywords: Two-phase systems, Electrolyte-resistance fluctuations, Contact angle, Collocation method, Gas evolution

*Corresponding author. Tel.: +33-1-44274148; fax: 33-1-44274074

E-mail address: francois.huet@upmc.fr(F. Huet)

1 in memoriam. 


\section{Introduction}

Formation of electrolytic bubbles is encountered in many industrial electrochemical applications because gas molecules are often generated from electrochemical reactions. This phenomenon has important implications because a part of the active electrode surface is screened by the attached bubbles, which affects charge, mass, and heat transfers at the electrode/electrolyte interface. The screening effect of electrolytic bubbles and the influence of the contact angle of attached bubbles, defined as the angle between the liquid-gas interface and the electrode surface, have been studied in several papers. For example, the modification of the primary distribution of potential and current lines (ohmic effects) due to the presence of a single spherical bubble attached to an infinite plane electrode has been analytically derived by Sides and Tobias and the incremental electrical resistance was calculated for a sparse array of spherical bubbles [1]. The electrode current density, which is quite negligible on a small area around the contact point, was found to approximately apply for nearly spherical bubbles having a contact angle lower than $17.5^{\circ}$. This analysis was later extended to bubbles making arbitrary contact angle with the electrode by Wilson and Hulme [2]. Dukovic and Tobias studied the effects of attached bubbles on the local distribution of current and on the potential drop at the electrode [3]. In addition to the increase in electrical resistance (ohmic effects), their model shows an increase in the surface overpotential due to the masked electrode area that increases the local current density, and a decrease in the concentration overpotential due to decreased local supersaturation near the bubbles. All these parameters have been shown to depend on the contact angle of the bubbles with the plane electrode.

The screening effect of attached electrolytic bubbles was also studied by Vogt et al. who introduced the bubble fractional coverage factor, defined as the area screened by the normal projection of the bubbles on the electrode surface. They showed this factor strongly depends on the electrolysis current density for both stagnant [4] and flowing [5-6] electrolytes. However, these authors neglected the influence of the contact angle when its value is lower than $90^{\circ}$ (wetting electrolyte), considering that the actual current density on the shaded area below the attached bubbles is small. 
Several authors have tried to take into account the influence of the contact angle on bubble growth rate, detachment diameter, and population density on the electrode, and to predict its dynamic variation at different stages of bubble evolution (nucleation, growth, and detachment). Trieu et al. studied hydrogen evolution formed by formaldehyde oxidation during electroless copper deposition on a vertical wall [7]. They theoretically showed the dependence of the side contact angle and bubble detachment radius and observed experimentally that the smaller the contact angle, the larger the evolving bubbles, which strongly affects the quality of the product plate.

Buehl and Westwater studied the effect of contact angle on the growth rate of bubbles in water supersaturated with carbon dioxide [8]. Their theoretical work and photographic observations of bubbles coming out from an artificial site drilled on a horizontal wall concluded that the effect of contact angle on bubble growth rates was small for contact angles between 0 and 90 degrees, even if the contact angle changed during bubble growth. Baum et al. studied the influence of the attachment of hydrogen bubbles produced during the etching of $\mathrm{Si}(100)$ in aqueous $\mathrm{KOH}$ on the surface roughness of microstructures [9]. Indeed, these bubbles are one of the causes of the formation of pyramids on the Si surface. They showed that the decrease in contact angle of the attached bubbles due to alcohol or oxidizing agent addition improves the surface finish.

Gas evolution may also be studied using the electrochemical noise technique, which consists in measuring the potential or current or electrolyte resistance fluctuations generated by the growth and detachment of bubbles, in order to derive the size and departure rate of bubbles. Despite the ability of the technique to monitor gas evolution in real time, very few studies have been reported in the literature on this topic. Gabrielli et al. showed that the mean departure rate, the mean radius of detaching bubbles, and the gas evolution efficiency can be derived from the spectral analysis of the potential or current fluctuations [10]. Hodgson showed that the influence of various electrocatalysts on the departure rate of chlorine bubbles evolving on ruthenium-titanium oxide anodes could be monitored by current noise measurements [11]. Compared to the analysis of the potential or current fluctuations, which depend on several factors (ohmic, activation, concentration of dissolved gas) 
$[3,12]$, the analysis of the fluctuations of the electrolyte resistance (ER) measured between the reference electrode and the working electrode is easier since only ohmic effects are concerned. Owing to the experimental set-up described in Ref. [13], Huet et al. simultaneously measured potential and electrolyte resistance (ER) fluctuations due to oxygen bubbles detaching from rough and porous horizontal $\mathrm{Ni}$ electrodes and showed that the size of detached bubbles depends on the electrode surface roughness [14]. Volanschi et al. studied the influence of various surfactants on the dynamic surface tension, which plays a significant role on the size and departure rate of evolving bubbles, from the simultaneous measurement of potential and ER on microcavity electrodes in aqueous solutions [15]. Benzaïd et al. also measured the ER fluctuations generated by hydrogen bubbles on cylindrical vertical carbon steel tensile specimens under cathodic polarisation [16]. However, their simplified model of the power spectral density of the ER fluctuations gave characteristic parameters of the gas evolution in only qualitative agreement with optical observations because of the complicated screening and dragging effects of rising bubbles for vertical electrodes.

In order to improve the theoretical models, it is necessary to quantify the effect of a bubble above or attached to a plane electrode of finite size on the ER. In a previous work, the ER increment due to the presence of an insulating sphere in contact with a disk electrode was calculated with the collocation mathematical method [17]. This configuration "single sphere - electrode" simulating a frozen bubble is the basic case in the study of gas evolution and can also find interest in other fields, such as when tracking the position of a moving spherical particle in a flow-channel [18]. However, only bubbles of contact angle equal to zero were concerned up to now. To extent the previous study, the influence of contact angle of an insulating sphere sitting at any position on a disk electrode is investigated in the present paper. 


\section{Theoretical treatment}

The mathematical collocation method used to calculate the distribution of the potential $\phi$ in the electrolyte when an insulating sphere simulating a bubble with a zero contact angle lies on a disk electrode has been detailed in Refs. [17,19] and is summarized in Appendix A. This method applies as well when the contact angle $\theta$ of the bubble is different from 0 . In that case, the bubble is simulated by a sphere cut off by a plane. Fig. 1 shows the notations used in this paper. The centre of the disk electrode of radius $a_{\mathrm{e}}$ is the origin $\mathrm{O}$ of the Cartesian coordinate system $(x, y, z)$ and the centre $O^{\prime}$ of the sphere of radius $a_{\mathrm{p}}$ corresponds to the point $\left(x_{0}, 0, z_{0}=a_{\mathrm{p}} \cos \theta\right)$, where $\theta$ is the contact angle of the truncated sphere, as defined in Fig. 1.

Once the coefficients $A_{\mathrm{nm}}$ and $C_{\mathrm{nm}}$ of the series expansion of the potential distribution in the electrolyte are determined (Appendix A), the ER can be calculated from the ratio of the potential $\phi_{0}$ applied to the surface of the electrode and the current $I$ flowing across the electrode, which is obtained by integrating the current density on the electrode $\left.\kappa(\partial \phi / \partial z)\right|_{z=0}$, where $\kappa$ is the electrolyte conductivity. The following expression of the ER is obtained for a sphere $(\theta=0)$ sitting on the electrode; it only depends on one coefficient $\left(A_{00}\right)$ of the series expansion of the potential, which is a purely imaginary complex number (see Appendix D in Ref. [17]):

$$
R_{\mathrm{e}}=\frac{\phi_{0}}{I}=\frac{\phi_{0}}{2 \pi \kappa a_{\mathrm{e}} j A_{00}}
$$

For a truncated sphere $(\theta \neq 0)$, the derivation of the ER is more involved. Indeed, there is no analytical expression of the current $I$ flowing across the electrode, in contrast with a perfect sphere, so that the current density must be numerically integrated on the electrode area not blocked by the sphere. When the truncated sphere is centred on the electrode axis, the expression of the ER depends on all coefficients $A_{\mathrm{n} 0}$ of the potential series expansion but the numerical calculations are relatively simple. When the sphere is not centred, these calculations are much more complex and some convergence problems arise, especially when the sphere approaches the electrode edge. The convergence of the calculations was ensured for $x_{0}<a_{\mathrm{e}}-a_{\mathrm{p}} \sin \theta$, that is, when the disk below the 
truncated sphere lies entirely on the electrode surface, at least for the largest spheres considered in this work $\left(a_{\mathrm{p}} / a_{\mathrm{e}}=0.4\right.$ or 0.5$)$.

The ER change due to the presence of the sphere was compared to the value of the theoretical ER of the disk electrode in the absence of the sphere, which is given by Newman's formula when the counter electrode is at infinity [20]:

$$
R_{\mathrm{e}, \mathrm{N}}=\frac{1}{4 \kappa a_{\mathrm{e}}}
$$

To compare the contribution of spheres of different sizes and located at various positions on the electrode, the ER variation was normalized:

$$
\Delta R_{\mathrm{e}, \text { norm }}=\frac{R_{\mathrm{e}}-R_{\mathrm{e}, \mathrm{N}}}{R_{\mathrm{e}, \mathrm{N}}}
$$

and the dimensionless radial position, $X_{0}$, of the sphere centre was introduced:

$$
X_{0}=\frac{x_{0}}{a_{\mathrm{e}}}
$$

\subsection{Case of a perfect sphere $(\theta=0)$}

As an example, Fig. 2 shows the normalized ER increment $\Delta R_{\mathrm{e}, \text { norm }}$ due to the presence of an insulating perfect sphere in contact with a disk electrode at any radial position $x_{0}$ on the diameter $y_{0}=0$ of the electrode [17]. The two peaks, which are obtained for small spheres $\left(a_{\mathrm{p}} / a_{\mathrm{e}}<1\right)$, are due to the fact that the current lines are more importantly blocked at the electrode edge $\left(X_{0}= \pm 1\right)$ where the local primary current density is higher. Despite the low values of $\Delta R_{\mathrm{e}, \text { norm }}$ (maximum of $0.3 \%$ for $a_{\mathrm{p}} / a_{\mathrm{e}}=0.1$ and $1.2 \%$ for $\left.a_{\mathrm{p}} / a_{\mathrm{e}}=0.2\right)$, the experimental values of $\Delta R_{\mathrm{e}, \text { norm }}$ measured in Ref. [17] were in excellent agreement with the calculated values in Fig. 2. 


\subsection{Case of a truncated sphere $(\theta \neq 0)$}

In a first part, the truncated sphere is positioned at the centre of the disk electrode $\left(x_{0}=0\right.$, $\left.y_{0}=0\right)$ where its influence on the ER is the lowest, according to Fig. 2 . The ER increment, $\Delta R_{\mathrm{e}, \text { norm, }}$ due to the truncated sphere is compared to that of a perfect sphere $(\theta=0)$ by introducing the ER increment in percentage, $\Delta R_{\mathrm{e}, \%}$, defined as follows :

$$
\Delta R_{\mathrm{e}, \%}\left(X_{0}=0, \theta\right)=\frac{\Delta R_{\mathrm{e}, \text { norm }}\left(X_{0}=0, \theta\right)-\Delta R_{\mathrm{e}, \text { norm }}\left(X_{0}=0,0\right)}{\Delta R_{\mathrm{e}, \text { norm }}\left(X_{0}=0,0\right)} \times 100
$$

Fig. 3 shows the values of $\Delta R_{\mathrm{e}, \text { norm }}$ and $\Delta R_{\mathrm{e}, \%}$ for five aspect ratios $a_{\mathrm{p}} / a_{\mathrm{e}}$ of the sphere and contact angles ranging between $0^{\circ}$ (perfect sphere) and $90^{\circ}$ (hemisphere). The $\Delta R_{\mathrm{e}, \text { norm }}$ values at the electrode centre show a significant dependence on the sphere size (less than $0.1 \%$ for $a_{\mathrm{p}} / a_{\mathrm{e}}=0.1$ and around $5.0 \%$ for $a_{\mathrm{p}} / a_{\mathrm{e}}=0.5$ ) and weaker variations with the contact angle. For small spheres $\left(a_{\mathrm{p}} / a_{\mathrm{e}} \leq 0.2\right), \Delta R_{\mathrm{e}, \%}$ is negative (for example, $-5.9 \%$ for $\theta=40^{\circ}$ and $a_{\mathrm{p}} / a_{\mathrm{e}}=0.1$ ) regardless of the contact angle. For larger spheres, $\Delta R_{\mathrm{e}, \%}$ is positive for small contact angles (for example, $+3.5 \%$ for $\theta=40^{\circ}$ and $\left.a_{\mathrm{p}} / a_{\mathrm{e}}=0.4\right)$ and negative when the contact angle increases $\left(\theta>62^{\circ}\right.$ for $\left.a_{\mathrm{p}} / a_{\mathrm{e}}=0.4\right)$. For an hemisphere $\left(\theta=90^{\circ}\right), \Delta R_{\mathrm{e}, \%}$ is negative whatever its size, with a much larger variation for small hemispheres (-43.4\% for $a_{\mathrm{p}} / a_{\mathrm{e}}=0.1$ against $-14.6 \%$ for $\left.a_{\mathrm{p}} / a_{\mathrm{e}}=0.5\right)$. Two opposing effects have to be considered to explain the results:

i) a surface effect: no current line can flow across the electrode surface under the truncated sphere, which increases the ER;

ii) a volume effect: the volume of a truncated sphere is smaller than that of a perfect sphere of same diameter, so that the current lines are modified on a smaller vertical distance; moreover, they rearrange more favourably around the foot of the sphere. Both effects decrease the ER.

For small truncated spheres, despite the fact that the surface area blocked under the sphere represents a large part of the projected surface of the sphere when it has a high contact angle $\left(\sin ^{2} \theta\right.$ $=41 \%$ for $\theta=40^{\circ}$ ), the $\Delta R_{\mathrm{e}, \%}$ value is less than that of a perfect sphere, indicating that the volume 
effect is dominant. This is because very few current lines reach the electrode below a perfect sphere when it is positioned at the centre of the electrode. The $\Delta R_{\mathrm{e}, \%}$ decrease with increasing $\theta$ observed for small spheres disappears for bigger spheres, indicating a more important surface effect, up to a limiting value of contact angle $\left(\theta=63^{\circ}\right.$ for $\left.a_{\mathrm{p}} / a_{\mathrm{e}}=0.4\right)$, from which the volume effect becomes dominant again. For the hemisphere $\left(\theta=90^{\circ}\right)$, the volume effect is clearly dominant for all spheres, the effect of the rearrangement of the current lines above the hemisphere being more important than that caused by the blocking of the electrode surface by the hemisphere.

Fig. 4 shows the values of $\Delta R_{\mathrm{e}, \text { norm }}$ and $\Delta R_{\mathrm{e}, \%}$ for truncated spheres of aspect ratio $a_{\mathrm{p}} / a_{\mathrm{e}}=0.2$ and 0.4 , which are now positioned at any radial position $X_{0}$ on the disk electrode. The different contact angles chosen correspond to those used in the experimental part presented below. As mentioned above, some convergence problems arose when the sphere approaches the electrode edge. The calculation of the potential distribution in the electrolyte was performed with two sets of degree $n$ and order $m$ and only results with very close values of $\Delta R_{\mathrm{e}, \text { norm }}$ were retained. No convergence problem could be observed for the largest sphere $\left(a_{\mathrm{p}} / a_{\mathrm{e}}=0.4\right.$ and $\theta=0^{\circ}, 41.4^{\circ}, 69.5^{\circ}$, and $\left.90^{\circ}\right)$ up to the limiting position $X_{0, \lim }=1-a_{\mathrm{p}} / a_{\mathrm{e}} \sin \theta$. For the smallest sphere $\left(a_{\mathrm{p}} / a_{\mathrm{e}}=0.2\right)$, for which the values of $\Delta R_{\mathrm{e}, \text { norm }}$ are very low, the convergence of the calculation was more difficult and only the results corresponding to the contact angles $0^{\circ}$ and $60^{\circ}$ are presented.

The $\Delta R_{\mathrm{e}, \text { norm }}$ values in Fig. $4 \mathrm{a}$ are obviously more important for the large sphere and increase with the distance $X_{0}$ to the electrode centre because the primary current density increases as well. For the hemisphere of aspect ratio 0.4 , the $\Delta R_{\mathrm{e}, \text { norm }}$ values are almost twice higher at the electrode edge than at the electrode centre. Fig. $4 \mathrm{~b}$ shows the $\Delta R_{\mathrm{e}, \%}$ values and allows the volume and surface effects identified above for a sphere positioned at the electrode centre to be compared. For the small sphere with a contact angle of $60^{\circ}$, the volume effect $\left(\Delta R_{\mathrm{e}, \%}<0\right)$ observed at the electrode centre is still predominant when the sphere is moved towards the electrode edge up to a position $X_{0}=0.7$ $\left(X_{0, \text { lim }}=0.83\right)$ where the influence of the blocking of the strong current lines by the truncated sphere 
becomes significant. For the bigger sphere $\left(a_{\mathrm{p}} / a_{\mathrm{e}}=0.4\right)$, the surface effect is preponderant at all positions $X_{0}$ of the sphere of contact angle $41.4^{\circ}$. In contrast, for the larger contact angles, the volume effect predominates when the sphere is located near the electrode centre while the surface effect dominates at a position $X_{0}>0.32\left(X_{0, \mathrm{lim}}=0.625\right)$ for the sphere of contact angle $69.5^{\circ}$ and $X_{0}>0.55\left(X_{0, \mathrm{lim}}=0.6\right)$ for the hemisphere.

\section{Experimental}

A glass crystallizing dish of $21 \mathrm{~cm}$ in diameter and $10 \mathrm{~cm}$ in height was used as an electrochemical cell (Fig. 5). The working electrode was the cross-section of a $1 \mathrm{~cm}$ in diameter stainless steel rod embedded in an epoxy resin and mounted flush in a polymethylmethacrylate holder. The electrolyte was a $0.1 \mathrm{M} \mathrm{Na}_{2} \mathrm{SO}_{4}$ solution prepared with deionised water. A large graphite foil flattened against the wall of the crystallizing dish was used as a counter electrode. A potential difference of $-300 \mathrm{mV}$ was applied between the working electrode and the counter electrode with a home-made potentiostat in a two-electrode cell configuration. The maximum DC current flowing through the cell was $2 \mu \mathrm{A}$. A sinusoidal voltage signal, $v_{\mathrm{hf}}$, of $100 \mathrm{kHz}$ and amplitude of $50 \mathrm{mV}$ was superimposed on the DC voltage of the potentiostat to measure the ER variations, $\Delta R_{\mathrm{e}}$. The sine wave current response, $v_{\mathrm{h} f} / R_{\mathrm{e}}$, at frequency $f_{\mathrm{hf}}$ was amplified, rectified with a diode, and low-pass filtered with a cut-off frequency of $10 \mathrm{~Hz}$, by using a home-made electronic device $[13,21]$. The amplitude of the voltage output signal, $v_{R_{\mathrm{e}}}$, obeyed the following relationship:

$$
v_{R_{\mathrm{e}}}=b \frac{1}{R_{\mathrm{e}}}+d
$$

The parameters $b$ and $d$ were determined through a calibration procedure in which the electrochemical cell was replaced by pure resistors. From Eq. 6 , the mean value of the ER, $R_{\mathrm{e}}$, could then be calculated from the mean value of $v_{R_{\mathrm{e}}}$ during the electrochemical experiments. For example, the ER mean value in the absence of sphere was $R_{\mathrm{e}}=33.8 \Omega$. In addition, the variations of the ER, $\Delta R_{\mathrm{e}}$, could be derived from the measured variations $\Delta v_{R_{\mathrm{e}}}$, with the equation: 


$$
\Delta v_{R_{\mathrm{e}}}=b \Delta\left(\frac{1}{R_{\mathrm{e}}}\right)=-\frac{b}{R_{\mathrm{e}}^{2}} \Delta R_{\mathrm{e}}
$$

obtained by differentiating Eq. 6 for ER variations of small amplitude.

Alumina spheres of 2 and $4 \mathrm{~mm}$ in diameter and excellent sphericity (Ceratec, The Netherlands) were used. Some of them were polished by the manufacturer to remove $0.1 \mathrm{~mm}, 0.5$ $\mathrm{mm}$, and $1 \mathrm{~mm}$ for the 2-mm-diameter spheres, and $0.5 \mathrm{~mm}, 1.3 \mathrm{~mm}$, and $2 \mathrm{~mm}$ for the 4-mmdiameter spheres, so as to obtain truncated spheres of different contact angles $\left(25.8^{\circ}, 60^{\circ}, 90^{\circ}\right.$ for the 2 -mm-diameter spheres of and $41.4^{\circ}, 69.5^{\circ}, 90^{\circ}$ for the 4-mm-diameter spheres). Using a stereo microscope, the spheres were carefully glued to the tip of a glass rod of about $100 \mu \mathrm{m}$ in diameter, as shown in the scanning electron microscope (SEM) images in Fig. 6 for a perfect sphere of $2 \mathrm{~mm}$ in diameter and a hemisphere of $4 \mathrm{~mm}$ in diameter. The experiments were performed at room temperature. The position of the sphere was controlled with a three-axis linear translation stage (Newport, VP-25XA) and driven by an ESP300 motion controller (Newport) allowing a $25 \mathrm{~mm}$ range motion of the glass rod holding the sphere with a $100 \mathrm{~nm}$ resolution in the three directions. Complementary information on the experimental set-up, measurement conditions and procedures may be found in Ref. [17].

In this work, the influence of the contact angle of the sphere on the ER was investigated for spheres located at the centre of the electrode because it was difficult to position the spheres far from the electrode axis at a very precise radial distance $X_{0}$, a parameter that has a strong influence on $\Delta R_{\mathrm{e}, \text { norm}}$, according to Fig. 2. Although the values of $\Delta R_{\mathrm{e}, \text { norm }}$ are lower at the electrode centre, they present a minimum at $X_{0}=0$ and the influence of $X_{0}$ could be negligible there. 


\section{Results and discussion}

\subsection{Case of perfect spheres}

Because of the elasticity of the glass rod, it was difficult to know exactly when the sphere was in contact with the disk electrode. The procedure described in Ref. [17] was used. Under visual control, the sphere was first positioned above the electrode centre and the parameters $x$ and $y$ giving the lateral position of the sphere were set to 0 . Then, the sphere was scanned above the electrode in the $x$ direction $(y=0)$ and the $v_{R_{\mathrm{e}}}$ variations were measured during forward and backward scans performed after progressively approaching the sphere to the disk electrode. As shown in Fig. 7a, the 2-mm-diameter sphere was in contact with the disk electrode since discrepancies can be observed in the $v_{R_{\mathrm{e}}}$ values of the first part of the backward scan and the second part of the forward scan. These discrepancies, due to the friction of the sphere with the electrode surface, disappeared when the sphere was raised at a height $h=3 \mu \mathrm{m}$ above the electrode (Fig. 7b), so that the forward and backward $v_{R_{\mathrm{e}}}$ curves overlapped quite well.

The coordinates of the sphere and the $v_{R_{\mathrm{e}}}$ variations must be converted to the dimensionless units presented in the theoretical section in order to compare the theoretical and experimental results. The parameters $X_{0}$ and $H$ give the normalized lateral position and the distance of the sphere above the electrode, respectively:

$$
X_{0}=\frac{x-x_{\mathrm{c}}}{a_{\mathrm{e}}} \quad \text { and } \quad H=\frac{h}{a_{\mathrm{e}}}=\frac{z-z_{\mathrm{c}}}{a_{\mathrm{e}}}
$$

where $x$ and $z$ are the lateral and vertical coordinates given by the translation stage while $x_{\mathrm{c}}$ and $z_{\mathrm{c}}$ are the coordinates of the electrode centre. On the other hand, the dimensionless increment of the ER due to the presence of the sphere, $\Delta R_{\mathrm{e}, \text { norm }}$, is derived from the $v_{R_{\mathrm{e}}}$ values and Eqs. 6 and 7:

$$
\Delta R_{\mathrm{e}, \text { norm }}=\frac{\Delta R_{\mathrm{e}}}{R_{\mathrm{e}}}=-\frac{\Delta v_{R_{\mathrm{e}}} / G}{v_{R_{\mathrm{e}}}-d}=\frac{\Delta v_{R_{\mathrm{e}}}}{B} \quad \text { with } \quad B=G\left(v_{R_{\mathrm{e}}}-d\right)
$$


where the factor $G$ is the gain of the amplifier used in some experiments to improve the measurement accuracy of $\Delta v_{R_{\mathrm{e}}}$, after elimination of the $v_{R_{\mathrm{e}}}$ DC component. Finally, to eliminate the DC level of about $0.48 \mathrm{~V}$ in Fig. 7 and take into account a possible tilt of the baseline (not observed in Fig. 7) due to a slight default in the horizontality of the electrode or to a linear drift in temperature that sometimes occurred, two new parameters, $\alpha$ and $\beta$, have been introduced in the following expression:

$$
\Delta R_{\mathrm{e}, \text { norm }}=\frac{v_{R_{\mathrm{e}}}-(\alpha x+\beta)}{B}
$$

In summary, for each scan of the sphere in contact with the electrode, the $\Delta R_{\mathrm{e}, \text { norm }}$ values were calculated from the raw $v_{R_{\mathrm{e}}}$ values after determining the four parameters $x_{\mathrm{c}}, B, \alpha$, and $\beta$ by fitting the $v_{R_{\mathrm{e}}}(x)$ experimental curve to the theoretical curve $\Delta R_{\mathrm{e}, \mathrm{norm}}\left(X_{0}, \theta=0\right)$ with the simplex algorithm.

In Fig. 7, the following values were measured: $v_{R_{\mathrm{e}}}=0.481 \mathrm{~V}, d=-33 \mathrm{mV}$, which gives a $B$ value of $0.514 \mathrm{~V}$ with $G=1$. Fig. 8 shows the fit obtained between the experimental curve of the forward scan presented in Fig. 7a and the theoretical curve $\Delta R_{\mathrm{e}, \text { norm }}\left(X_{0}, \theta=0\right)$ presented in Fig. 2 for the sphere size $a_{\mathrm{p}} / a_{\mathrm{e}}=0.2$. The excellent agreement between the two curves, obtained with a fitted value $B=0.543 \mathrm{~V}$ close to the expected value (relative error of $5.6 \%$ ), validates the methodology used for measuring small variations of $\Delta R_{\mathrm{e}, \text { norm }}$ (less than $1.2 \%$ ) during the scan of the sphere.

\subsection{Case of truncated spheres}

The above method to ensure the contact between the sphere and the electrode was not appropriate for truncated spheres. Indeed, the contact area on the electrode is much greater than for perfect spheres, so that the glass rod broke during the forward and backward scans of the truncated spheres because of the important friction and a possible inclination of the electrode surface. A new method had to be developed in this work. The truncated sphere was firstly positioned above the electrode centre by measuring the $v_{R_{\mathrm{e}}}$ signal during successive scans of the sphere in the $\mathrm{O} x$ and $\mathrm{O} y$ 
directions without touching the electrode. The minimum value of $v_{R_{\mathrm{e}}}$ corresponds to the electrode centre above which the truncated sphere was placed before being moved down toward the electrode. Fig. 9a presents the $v_{R_{\mathrm{e}}}$ variations as a function of the vertical position $z$ during the descent and rise of a 2-mm-diameter sphere above the electrode centre ( $z$ decreased when the sphere went down). The two curves are almost identical, the slight differences coming probably from slight variations in the electrolyte temperature during the experiment. The ER increased when the sphere approached the electrode because the current lines were more strongly deviated under the sphere. When the sphere touched the electrode, the $v_{R_{\mathrm{e}}}$ signal became erratic while the glass rod was still going down ( $z$ decreased) without breaking because of its elasticity. This is not obvious in the enlarged view of the approach curve shown in the inset of Fig. 9a because of the fluctuations observed on this curve. However, it will be clearer on the approach curve of truncated spheres, as shown below, and this will allow the vertical position $z_{\mathrm{c}}$ at which the sphere was in contact with the disk electrode to be determined. Since the disk electrode and the plane of the truncated sphere were polished and because the elasticity of the glass rod could force the whole plane surface of the truncated sphere to touch the disk electrode, the quality of the contact between the sphere and the electrode was considered to be good. It must be noticed that this method is the only one that can be used for truncated spheres that cannot be scanned in contact with the electrode without breaking the glass rod. Also, performing measurements at only one point of the electrode, here the electrode centre, gives the advantage of being less dependent of the horizontality of the electrode surface. At last, there is no need to position the sphere exactly above the electrode centre before measuring the approach curve since the $v_{R_{\mathrm{e}}}(x)$ curve passes through a minimum at the electrode centre.

Fig. $9 \mathrm{~b}$ shows the fitting of the average of the approach and backward curves of Fig.9a with the theoretical normalized ER increment $\Delta R_{\mathrm{e}, \text { norm }}$ as a function of the normalized height $H$ above the electrode disk. In this example, the measured values, $v_{R_{\mathrm{e}}}=0.195 \mathrm{~V}$ and $d=-27 \mathrm{mV}$, give a $B$ value of $22.64 \mathrm{~V}$ with $G=102$, while the fitted values were $z_{\mathrm{c}}=-10.362 \mathrm{~mm}, \alpha=6.3 \mu \mathrm{V} / \mathrm{mm}, \beta=25.8$ 
$\mathrm{mV}$, and $B=22.82 \mathrm{~V}$. This $B$ value was close to the expected value (relative error of $0.8 \%$ ). This, together with the excellent agreement between the experimental and theoretical curves, validates the measurement of the low values of $\Delta R_{\mathrm{e}, \text { norm }}$ (less than $0.5 \%$ ) when the sphere approaches the electrode.

The influence of the contact angle of the sphere on the ER is now analyzed. Fig. 10a presents the $v_{R_{\mathrm{e}}}$ variations induced by the vertical approach of a 2-mm-diameter sphere $\left(a_{\mathrm{p}} / a_{\mathrm{e}}=0.2\right)$ of contact angle $0^{\circ}$ (sphere), $25.8^{\circ}, 60^{\circ}$, and $90^{\circ}$ (hemisphere) above the electrode centre. The $z$ distance corresponds to an arbitrary origin of the positioning device so that the contact point on the electrode did not correspond to the same $z$ value for each experiment. Fig. 10b shows the normalized ER increment $\Delta R_{\mathrm{e}, \text { norm }}$ as a function of the dimensionless height $H$ of the sphere. These curves were calculated from the raw data in Fig. 10a, using Eq. 9 with a $B$ value of $22.82 \mathrm{~V}$ and Eq. 8 to derive $H$ from the $z$ and $z_{\mathrm{c}}$ values. It should be noticed that the theoretical approach curve was not available for the truncated spheres to fit the experimental $v_{R_{\mathrm{e}}}(\mathrm{z})$ curve and determine the vertical position $z_{\mathrm{c}}$ where the sphere touches the electrode. As explained above, $z_{\mathrm{c}}$ can be taken as the position where the $v_{R_{\mathrm{e}}}$ signal became erratic at the time the sphere touched the electrode while the glass rod was still going down because its elasticity. This is clearly shown in the inset of Fig. 10a for the hemisphere. The distance between two successive $z$ values in the approach curve was 25 $\mu \mathrm{m}$ so that the error on $z_{\mathrm{c}}$ could be estimated to be about $10 \mu \mathrm{m}$.

Fig. $10 \mathrm{~b}$ shows that when a sphere of any contact angle was far above the electrode centre $(H>2), \Delta R_{\mathrm{e}, \text { norm }}$ was close to 0 . In other words, a sphere of $2 \mathrm{~mm}$ in diameter $\left(a_{\mathrm{p}} / a_{\mathrm{e}}=0.2\right)$ located at a height twice larger than the electrode radius $(5 \mathrm{~mm})$ has no influence on the ER. When the sphere is in contact with the electrode centre, the $\Delta R_{\mathrm{e}, \text { norm }}$ value was low, ranging from $0.27 \%$ to $0.42 \%$ depending on the contact angle of the sphere. It must be noted that the maximum value of $\Delta R_{\mathrm{e}, \text { norm }}$ did not correspond to the contact position at the electrode centre; it was obtained when the sphere was slightly above the electrode centre, for example at a distance $H_{\max } \approx 0.1$ for the 
truncated sphere of contact angle $60^{\circ}$ and $H_{\max } \approx 0.13$ for the hemisphere. For perfect spheres, the effect is much less pronounced but it was already observed in Ref. [19] (the down-pointing arrow in Fig. 3 shows the maximum of resistance increment). This phenomenon can be explained by the rearrangement of the current lines according to the position of the sphere. When a truncated sphere of high contact angle is located slightly above the electrode, the electrode surface under the sphere is not very active because very few current lines can reach the electrode surface through the thin electrolyte layer below the sphere, so that the surface effect on the ER is almost the same as when the sphere touches the electrode. In contrast, the volume of conductive electrolyte above the sphere that has been replaced by the insulating sphere and the rearrangement of the current lines around it lead to an increase in the ER (volume effect). At a distance higher than $H_{\max }$, the electrode surface under the sphere becomes more active because the current lines can reach the electrode more easily. The surface effect on the ER decreases while the volume effect is not changed, which explains the decrease in ER observed. The $\Delta R_{\mathrm{e}, \text { norm }}$ maximum value was observed at lower values of $H_{\max }$ for the truncated spheres of small contact angle (for example, $H_{\max } \approx 0.04$ for $\theta=25.8^{\circ}$ ), and this maximum value was much closer to that at $H=0$, because the electrode surface screened by the sphere was narrower, which limited the rearrangement of the current lines when the sphere was moved slightly above the electrode centre.

The results concerning the 4-mm-diameter $\left(a_{\mathrm{p}} / a_{\mathrm{e}}=0.4\right)$ truncated spheres of contact angle $41.4^{\circ}, 69.5^{\circ}$, and $90^{\circ}$ are presented in Fig. 11 . The $v_{R_{\mathrm{e}}}$ variations induced by the vertical approach of the sphere above the electrode centre were measured with an amplifier of gain $G=10$ and are given in Fig. 11a while Fig. 11b shows the normalized ER increment $\Delta R_{\mathrm{e}, \text { norm }}$ as a function of the

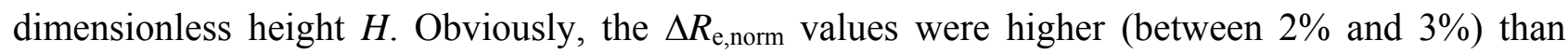
those of the 2-mm-diameter spheres because of a more important screening of the electrode surface. As for the smaller spheres, the maximum values of $\Delta R_{\mathrm{e}, \text { norm }}$ were obtained when the spheres were slightly above the electrode $\left(H_{\max } \approx 0.09\right.$ for $\theta=69.5^{\circ}$ and $H_{\max } \approx 0.15$ for the hemisphere). 
To compare the theoretical and experimental results, the experimental values of $\Delta R_{\mathrm{e}, \text { norm }}\left(X_{0}=0, \theta\right)$ for each truncated sphere of contact angle $\theta$ placed at the electrode centre were used to determine the ER increment in percentage, $\Delta R_{\mathrm{e}, \%}$, of the $\Delta R_{\mathrm{e}, \text { norm }}\left(X_{0}=0,0^{\circ}\right)$ value for a perfect sphere also placed at the electrode centre (Eq. 5). Table 1 summarizes the theoretical and experimental results for the spheres of $2 \mathrm{~mm}$ and $4 \mathrm{~mm}$ in diameter in contact with the electrode centre $(H=0)$ and Fig. 12 presents the theoretical variations of $\Delta R_{\mathrm{e}, \%}$ as a function of the contact angle (Fig. 3b) and the experimental $\Delta R_{\mathrm{e}, \%}$ values given in Table 1 . The excellent agreement between the theoretical and experimental values allows validating the methodologies used in this work, both in the modelling part and in the experimental measurements.

\section{Conclusions}

This paper is an extension of previous works aimed at improving the analysis of the electrolyte resistance (ER) fluctuations generated by spherical insulating entities (gas bubbles, droplets, solid particles, etc.) passing close or attached to a disk electrode. In the study of gasevolving electrodes, the contact angle of bubbles with the electrode surface plays an important role on the size of the evolving bubbles. In the present work, the influence of the contact angle of spheres on the ER is investigated.

The mathematical collocation method allowed determining the ER increment due to the presence of a single sphere of any contact angle located at any position on the surface of a disk electrode. Low values of the ER increment were found for a sphere located at the electrode centre,

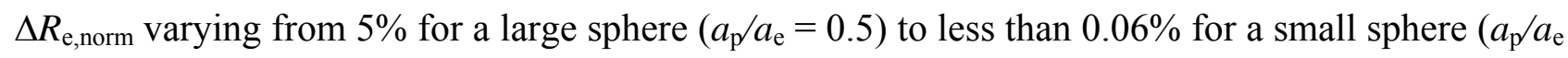
$=0.1$. These values increased by a factor less than 2 that depends on the contact angle when the sphere was moved towards the electrode edge. For the sphere sizes investigated, the ER increment of a truncated sphere, $\Delta R_{\mathrm{e}, \%}$, varied between $-45 \%$ and $+35 \%$ of the ER increment due to a perfect sphere. These variations, which depend on the size of the sphere, its lateral position on the electrode, and its contact angle, could be explained by the interplay of two competing effects 
influencing the ER: a surface effect related to the accessibility of the electrode surface below the sphere to the current lines, and a volume effect related to the smaller volume of the insulating truncated sphere.

Owing to a high-precision motorized translation stage and a specific low-noise ER measurement device, the small ER increments due to the presence of single spheres of different contact angle located at the electrode centre could be experimentally determined. An excellent agreement was found between the experimental and theoretical results. 


\section{Appendix A. Calculation of the potential distribution}

The calculation of the potential primary distribution in the electrolyte, when an insulating sphere is sitting on a disk electrode, is based on the integration of Laplace's equation $\left(\nabla^{2} \phi=0\right)$, where $\phi$ is the electrical potential in the solution, when the concentration and temperature of the electrolyte, and therefore its conductivity, are uniform. The boundary conditions are:

a) $\phi$ is equal to 0 at any point infinitely far from the electrode

b) $\phi$ is equal to the applied potential $\phi_{0}$ on the disk electrode surface

c) the normal component of the current is equal to 0 at any point of the sphere surface and of the insulating plane; as a consequence, the normal component of the potential gradient $\partial \phi / \partial \mathrm{n}$ is equal to 0 on these surfaces.

This problem can be solved using the mathematical collocation method. In this approach, the general solution of Laplace's equation is written as:

$$
\phi=\phi_{\mathrm{e}}+\phi_{\mathrm{p}}
$$

where $\phi_{\mathrm{e}}$ represents the potential in the coordinate system related to the electrode (oblate spheroidal coordinates $\xi_{\mathrm{e}}, \eta_{\mathrm{e}}, \varphi_{\mathrm{e}}$, with the origin at the centre of the electrode) and $\phi_{\mathrm{p}}$ represents the potential in the coordinate system related to the sphere (spherical coordinates $\rho_{p}, \eta_{p}, \varphi_{p}$ with the origin at the centre of the sphere). It can be shown [17], after introducing the concept of "double-sphere" (a second sphere of radius $a_{\mathrm{p}}$ and centre $\mathrm{O}^{\prime \prime}\left(x_{0}, 0,-z_{0}\right)$ is added), that $\phi$ can be written as the sum of base functions:

$$
\begin{aligned}
& \phi(x, y, z)=\phi_{\mathrm{e}}+\phi_{\mathrm{p}}=\sum_{n=0}^{+\infty} \sum_{\substack{m=0 \\
n-m \text { even }}}^{n} A_{\mathrm{nm}}\left(Q_{\mathrm{n}}^{\mathrm{m}}\left(j \sinh \xi_{\mathrm{e}}\right) P_{\mathrm{n}}^{\mathrm{m}}\left(\cos \eta_{\mathrm{e}}\right) \cos \left(m \varphi_{\mathrm{e}}\right)\right) \\
& +\sum_{n=0}^{+\infty} \sum_{m=0}^{n} C_{\mathrm{nm}}\left(\rho_{\mathrm{p} 1}^{-\mathrm{n}-1} P_{\mathrm{n}}^{\mathrm{m}}\left(\cos \eta_{\mathrm{p} 1}\right) \cos \left(m \varphi_{\mathrm{p} 1}\right)+\rho_{\mathrm{p} 2}^{-\mathrm{n}-1} P_{\mathrm{n}}^{\mathrm{m}}\left(\cos \eta_{\mathrm{p} 2}\right) \cos \left(m \varphi_{\mathrm{p} 2}\right)\right)
\end{aligned}
$$

where $j$ is the square root of -1 and $P_{\mathrm{n}}^{\mathrm{m}}$ and $Q_{\mathrm{n}}^{\mathrm{m}}$ are the Legendre functions of degree $n$ and order $m(m \leq n)$ of the first and second kind, respectively. This solution satisfies the simplest boundary 
conditions at infinity, Eq. (A1), and on the insulating plane, Eq. (A3). The coefficients $A_{\mathrm{nm}}$ and $C_{\mathrm{nm}}$ of the series expansion must be determined so that the boundary conditions on the metallic electrode, Eq. (A2), and on the insulating sphere, Eq. (A3), are satisfied. The method consists in choosing a certain number of points on the sphere and on the electrode and enforcing the boundary conditions at these points on the electrode:

$$
\begin{aligned}
& \sum_{n=0}^{+\infty} \sum_{\substack{m=0 \\
n-m}}^{n} A_{\mathrm{nm}}\left(Q_{\mathrm{n}}^{\mathrm{m}}\left(j \sinh \xi_{\mathrm{e}}\right) P_{\mathrm{n}}^{\mathrm{m}}\left(\cos \eta_{\mathrm{e}}\right) \cos \left(m \varphi_{\mathrm{e}}\right)\right) \\
& \quad+\sum_{n=0}^{+\infty} \sum_{m=0}^{n} C_{\mathrm{nm}}\left(\rho_{\mathrm{p} 1}^{-\mathrm{n}-1} P_{\mathrm{n}}^{\mathrm{m}}\left(\cos \eta_{\mathrm{p} 1}\right) \cos \left(m \varphi_{\mathrm{p} 1}\right)+\rho_{\mathrm{p} 2}^{-\mathrm{n}-1} P_{\mathrm{n}}^{\mathrm{m}}\left(\cos \eta_{\mathrm{p} 2}\right) \cos \left(m \varphi_{\mathrm{p} 2}\right)\right)=\phi_{0}
\end{aligned}
$$

and on the sphere:

$$
\begin{aligned}
& \sum_{n=0}^{+\infty} \sum_{\substack{m=0 \\
n-m}}^{n} A_{\mathrm{nm}} \frac{\partial}{\partial n}\left(Q_{\mathrm{n}}^{\mathrm{m}}\left(j \sinh \xi_{\mathrm{e}}\right) P_{\mathrm{n}}^{\mathrm{m}}\left(\cos \eta_{\mathrm{e}}\right) \cos \left(m \varphi_{\mathrm{e}}\right)\right) \\
& \quad+\sum_{n=0}^{+\infty} \sum_{m=0}^{n} C_{\mathrm{nm}} \frac{\partial}{\partial n}\left(\rho_{\mathrm{p} 1}^{-\mathrm{n}-1} P_{\mathrm{n}}^{\mathrm{m}}\left(\cos \eta_{\mathrm{p} 1}\right) \cos \left(m \varphi_{\mathrm{p} 1}\right)+\rho_{\mathrm{p} 2}^{-\mathrm{n}-1} P_{\mathrm{n}}^{\mathrm{m}}\left(\cos \eta_{\mathrm{p} 2}\right) \cos \left(m \varphi_{\mathrm{p} 2}\right)\right)=0
\end{aligned}
$$

The calculation of the coefficients $A_{\mathrm{nm}}$ and $C_{\mathrm{nm}}$ was performed with maximum values of degree $n$ and order $m, n_{\max }$ and $m_{\max }$, typically around 50 and 35 , respectively. In that case, the number of points on the electrode and on the sphere was about three times the number of values $A_{\mathrm{nm}}$ and $C_{\mathrm{nm}}$ to be determined. Obviously, only a finite number of coefficients $A_{\mathrm{nm}}$ and $C_{\mathrm{nm}}$ were determined (see Appendix C in Ref. [17]). The potential $\phi$ could then be calculated at any point $(x, y, z)$ in the solution.

For a truncated sphere (contact angle $\theta$ different from 0 ), the expression of the potential distribution (Eq. A5) is still valid if the angles $\eta_{\mathrm{p} 1}$ et $\eta_{\mathrm{p} 2}$ are now ranging between 0 and $\pi-\theta$ instead of 0 and $\pi$. The coefficients $A_{\mathrm{nm}}$ and $C_{\mathrm{nm}}$ are still calculated with the boundary conditions on the electrode (Eq. A6) and on the sphere (Eq. A7), the points on the sphere and on the electrode being obviously limited to the zone in contact with the electrolyte $(\eta \leq \pi-\theta)$. 


\section{References}

[1] P.J. Sides, C.W. Tobias, J. Electrochem. Soc. 127 (1980) 288.

[2] S.D.R. Wilson, A. Hulme, Proc. R. Soc. Lond. A 387 (1983) 133.

[3] J. Dukovic, C.W. Tobias, J. Electrochem. Soc. 134 (1987) 331.

[4] H. Vogt, R.J. Balzer, Electrochim. Acta 50 (2005) 2073.

[5] J. Eigeldinger, H. Vogt, Electrochim. Acta 45 (2000) 4449.

[6] R.J. Balzer, H. Vogt, J. Electrochem. Soc. 150 (2003) E11.

[7] H. Trieu, R. Chandran, J. Electrochem. Soc., 136 (1989) 2218.

[8] W. M. Buehl, J. W. Westwater, A.I.Ch.E. Journal, 12 (1966) 571.

[9] T. Baum, J. Satherley, D. J. Schiffrin, Langmuir, 14 (1998) 2925.

[10] C. Gabrielli, F. Huet, M. Keddam, A. Sahar, J. Applied Electrochem. 19 (1989) 683.

[11] D. R. Hodgson, Electrochim. Acta, 41 (1996) 605.

[12] A. Benzaïd, F. Huet, M. Jérôme, F. Wenger, C. Gabrielli, J. Galland, Electrochim. Acta 47 (2002) 4325.

[13] C. Gabrielli, F. Huet, M. Keddam, J. Electrochem. Soc. 138 (1991) L82.

[14] F. Huet, M. Musiani, R. Nogueira, J. Solid State Electrochem. 8 (2004) 786.

[15] A. Volanschi, W. Olthuis, P. Bergveld, Sensor. Actuat. A 52 (1996) 18.

[16] A. Benzaïd, F. Huet, M. Jérôme, F. Wenger, C. Gabrielli, J. Galland, Electrochim. Acta 47 (2002) 4315 .

[17] H. Bouazaze, J. Fransaer, F. Huet, P. Rousseau, V. Vivier, Electrochim. Acta, 55 (2010) 1645.

[18] N.V. Rees, S.M. Matthews, K. Yunus, A.C. Fisher, R.G. Compton, Angew. Chem. Int. Ed. 48 (2009) 2376.

[19] J. Fransaer, J. P. Celis, J. R. Roos, J. Electroanal. Chem., 391 (1995) 11.

[20] J. Newman, J. Electrochem. Soc. 113 (1966) 501.

[21] V. Bouet, J. Fransaer, F. Huet, G. Maurin, J.P. Célis, J. Electrochem. Soc. 145 (1998) 436. 


\section{Figure and Table Captions}

Table 1 Theoretical and experimental values of $\Delta R_{\mathrm{e}, \text { norm }}$ and $\Delta R_{\mathrm{e}, \%}$ as a function of the contact angle of the spheres of $2 \mathrm{~mm}$ and $4 \mathrm{~mm}$ in diameter in contact with the electrode centre.

Fig. 1. Schematic drawing of the truncated sphere in contact with the electrode.

Fig. 2. Normalized ER increment $\Delta R_{\mathrm{e}, \text { norm }}$ due to the presence of an insulating sphere in contact with the electrode as a function of the radial position $X_{0}$ for 2 sphere sizes $\left(a_{\mathrm{p}} / a_{\mathrm{e}}=0.1\right.$ and 0.2). [17]

Fig. 3. Normalized ER increment $\Delta R_{\mathrm{e}, \text { norm }}$ (a) and $\Delta R_{\mathrm{e}, \%}$ (b) as a function of the contact angle of the insulating sphere of various size positioned at the electrode centre.

Fig. 4. Normalized ER increment $\Delta R_{\mathrm{e}, \text { norm }}$ (a) and $\Delta R_{\mathrm{e}, \%}$ (b) due to the presence of an insulating sphere of various contact angle and size $\left(a_{\mathrm{p}} / a_{\mathrm{e}}=0.2\right.$ and 0.4$)$ as a function of its radial position $X_{0}$.

Fig. 5. Experimental set-up used for measuring the ER variations due to the presence of a sphere shifted above or in contact with the electrode.

Fig. 6. SEM images of the sphere of diameter $2 \mathrm{~mm}$ (a) and hemisphere $\left(\theta=90^{\circ}\right)$ of diameter 4 $\mathrm{mm}(\mathrm{b})$.

Fig. 7. Variation of $v_{R_{\mathrm{e}}}$ as a function of the radial position $x$ of an insulating 2-mm-diameter sphere plane (a) in contact with $(h=0)$ and $(\mathrm{b})$ above $(h=3 \mu \mathrm{m})$ a 10 -mm-diameter stainless steel electrode during a forward and backward scan at a $0.4 \mathrm{~mm} / \mathrm{s}$ rate.

Fig. 8. Theoretical and experimental normalized $\Delta R_{\mathrm{e}, \text { norm }}$ variations as a function of the normalized radial position $X_{0}$ of an insulating 2-mm-diameter sphere in contact with the electrode. The experimental data correspond to the forward scan in Fig. 7a.

Fig. 9. (a) Variation of $v_{R_{\mathrm{e}}}$ as a function of the vertical position $z$ of an insulating 2-mm-diameter sphere above the electrode centre for an approach and backward scan at a $0.2 \mathrm{~mm} / \mathrm{s}$ rate; 
(b) experimental normalized $\Delta R_{\mathrm{e}, \text { norm }}$ variations (average of the approach and backward curves) fitted with the theoretical curve, as a function of the normalized vertical position $H$ of the sphere above the electrode.

Fig. 10. (a) Variation of $v_{R_{\mathrm{e}}}$ as a function of the vertical position $z$ and (b) normalized ER increment $\Delta R_{\mathrm{e}, \text { norm }}$ as a function of the normalized height $H$ of insulating 2-mm-diameter spheres with different contact angles above the electrode centre $(0.2 \mathrm{~mm} / \mathrm{s}$ scan rate $)$.

Fig. 11. (a) Variation of $v_{R_{\mathrm{e}}}$ as a function of the vertical position $z$ and (b) normalized ER increment $\Delta R_{\mathrm{e}, \text { norm }}$ as a function of the normalized height $H$ of insulating 4-mm-diameter spheres with different contact angles above the electrode centre $(0.2 \mathrm{~mm} / \mathrm{s}$ scan rate $)$.

Fig. 12. Theoretical and experimental normalized ER increment $\Delta R_{\mathrm{e}, \%}$ as a function of the contact angle of the spheres of $2 \mathrm{~mm}$ and $4 \mathrm{~mm}$ in diameter in contact with the electrode centre. 
Table 1 Theoretical and experimental values of $\Delta R_{\mathrm{e}, \text { norm }}$ and $\Delta R_{\mathrm{e}, \%}$ as a function of the contact angle of the spheres of $2 \mathrm{~mm}$ and $4 \mathrm{~mm}$ in diameter in contact with the electrode centre.

\begin{tabular}{l|c|c|c|c|c|c|c|c}
\hline & \multicolumn{3}{|c|}{$\left(a_{\mathrm{p}} / a_{\mathrm{e}}=0.2\right)$} & \multicolumn{5}{c}{$\left(a_{\mathrm{p}} / a_{\mathrm{e}}=0.4\right)$} \\
\hline Contact angle / degree & 0 & 25.8 & 60 & 90 & 0 & 41.4 & 69.5 & 90 \\
\hline Theoretical $\Delta R_{\mathrm{e}, \text { norm }, \%}$ & 0.425 & 0.420 & 0.372 & 0.256 & 2.84 & 2.94 & 2.71 & 2.10 \\
\hline Experimental $\Delta R_{\mathrm{e}, \text { norm }} \%$ & 0.424 & 0.414 & 0.369 & 0.267 & 2.85 & 2.94 & 2.73 & 2.17 \\
\hline Theoretical $\Delta R_{\mathrm{e}, \%} \%$ & 0 & -1.1 & -12.5 & -39.8 & 0 & +3.5 & -4.6 & -26 \\
\hline Experimental $\Delta R_{\mathrm{e}, \%} \%$ & +0.2 & -2.6 & -13.2 & -37.2 & +0.4 & +3.5 & -3.9 & -24 \\
\hline
\end{tabular}




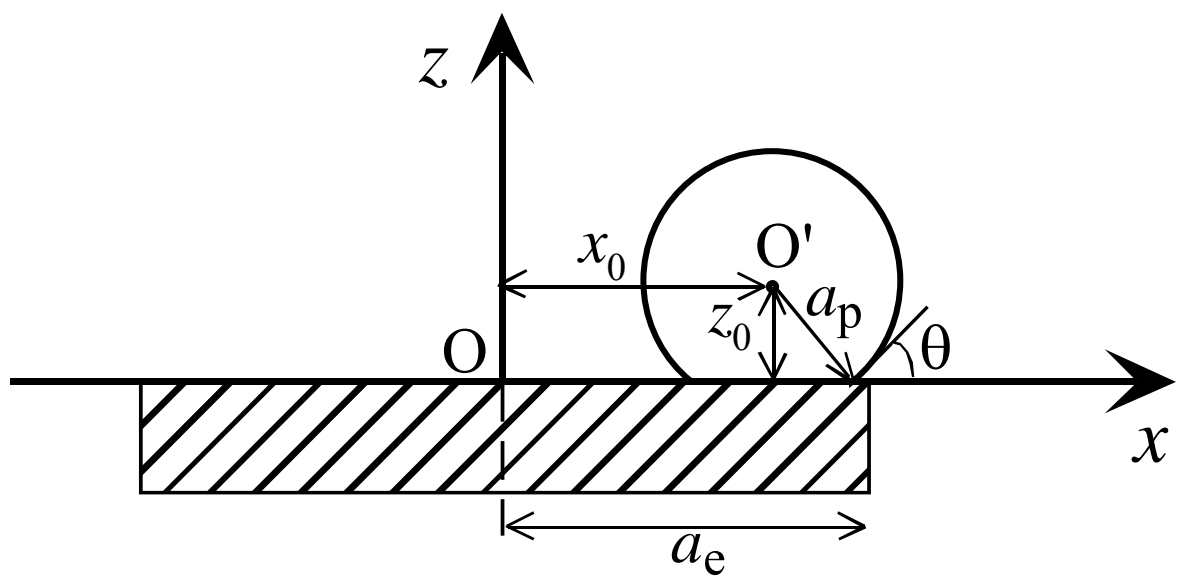

Fig. 1. Schematic drawing of the truncated sphere in contact with the electrode. 


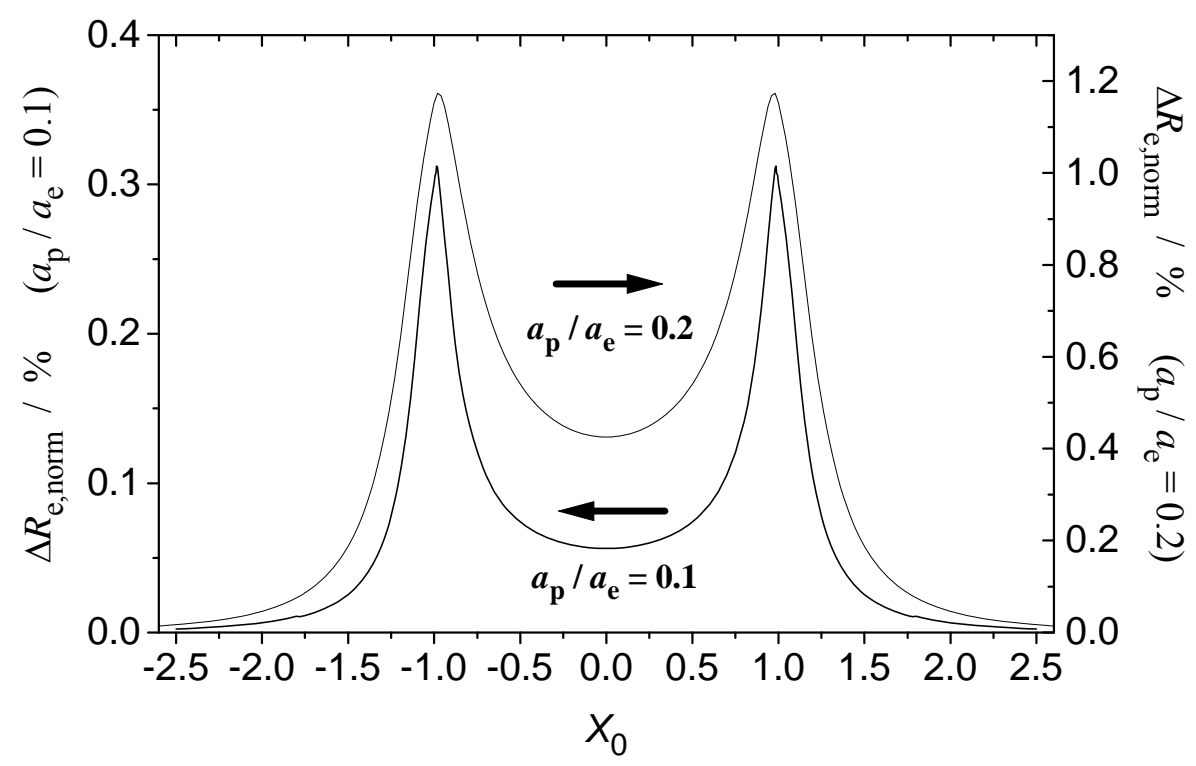

Fig. 2. Normalized ER increment $\Delta R_{\mathrm{e}, \text { norm }}$ due to the presence of an insulating sphere in contact with the electrode as a function of the radial position $X_{0}$ for 2 sphere sizes $\left(a_{\mathrm{p}} / a_{\mathrm{e}}=0.1\right.$ and 0.2). [17] 
(a)

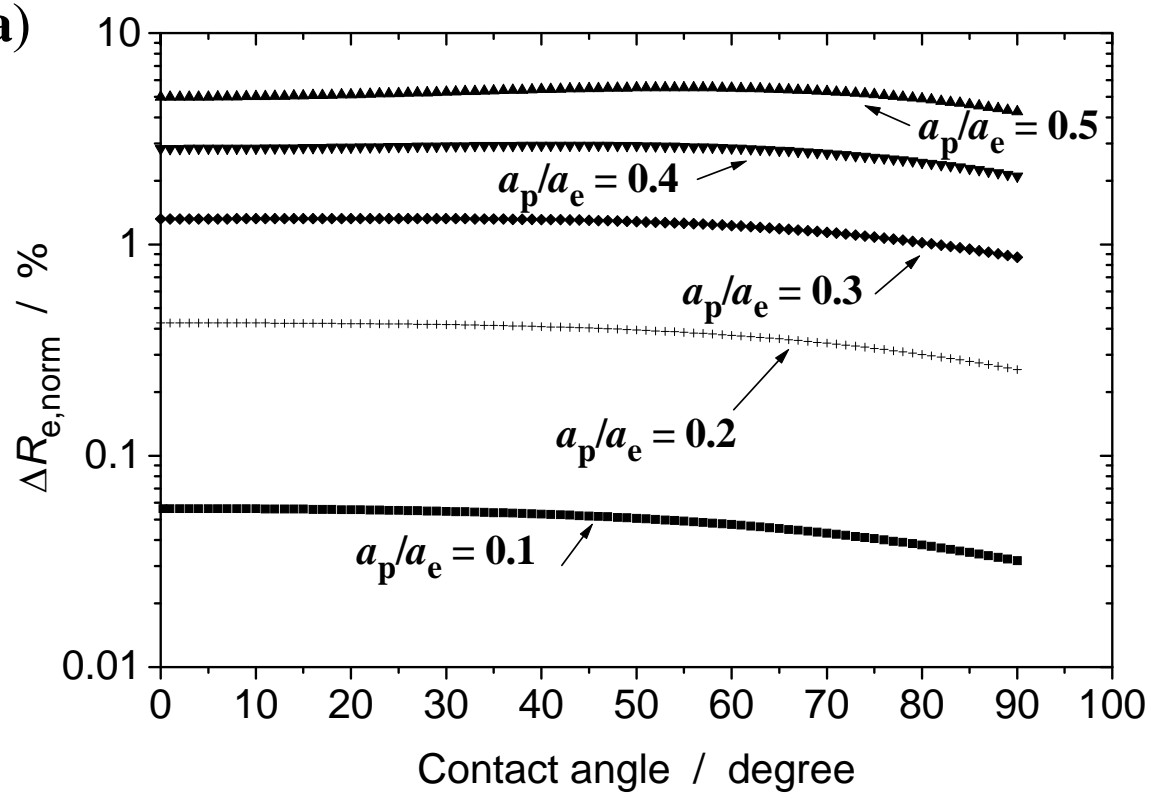

(b)

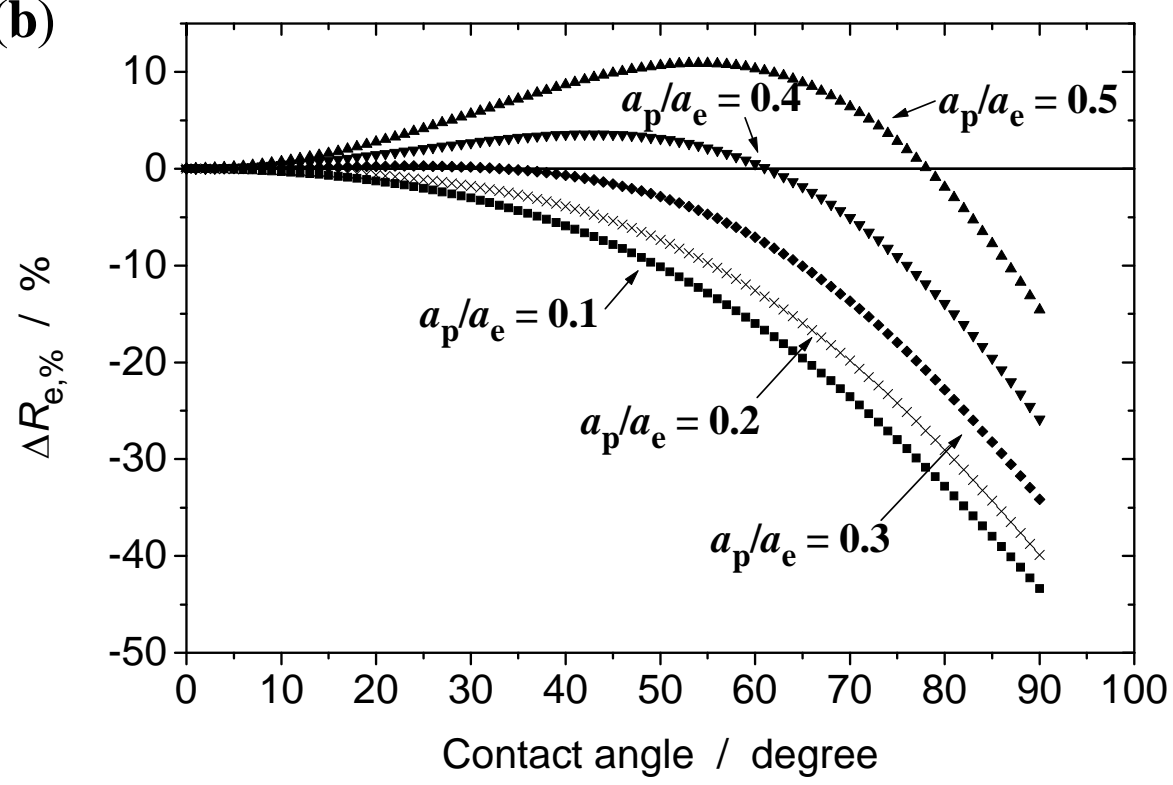

Fig. 3. Normalized ER increment $\Delta R_{\mathrm{e}, \text { norm }}$ (a) and $\Delta R_{\mathrm{e}, \%}$ (b) as a function of the contact angle of the insulating sphere of various size positioned at the electrode centre. 
(a)
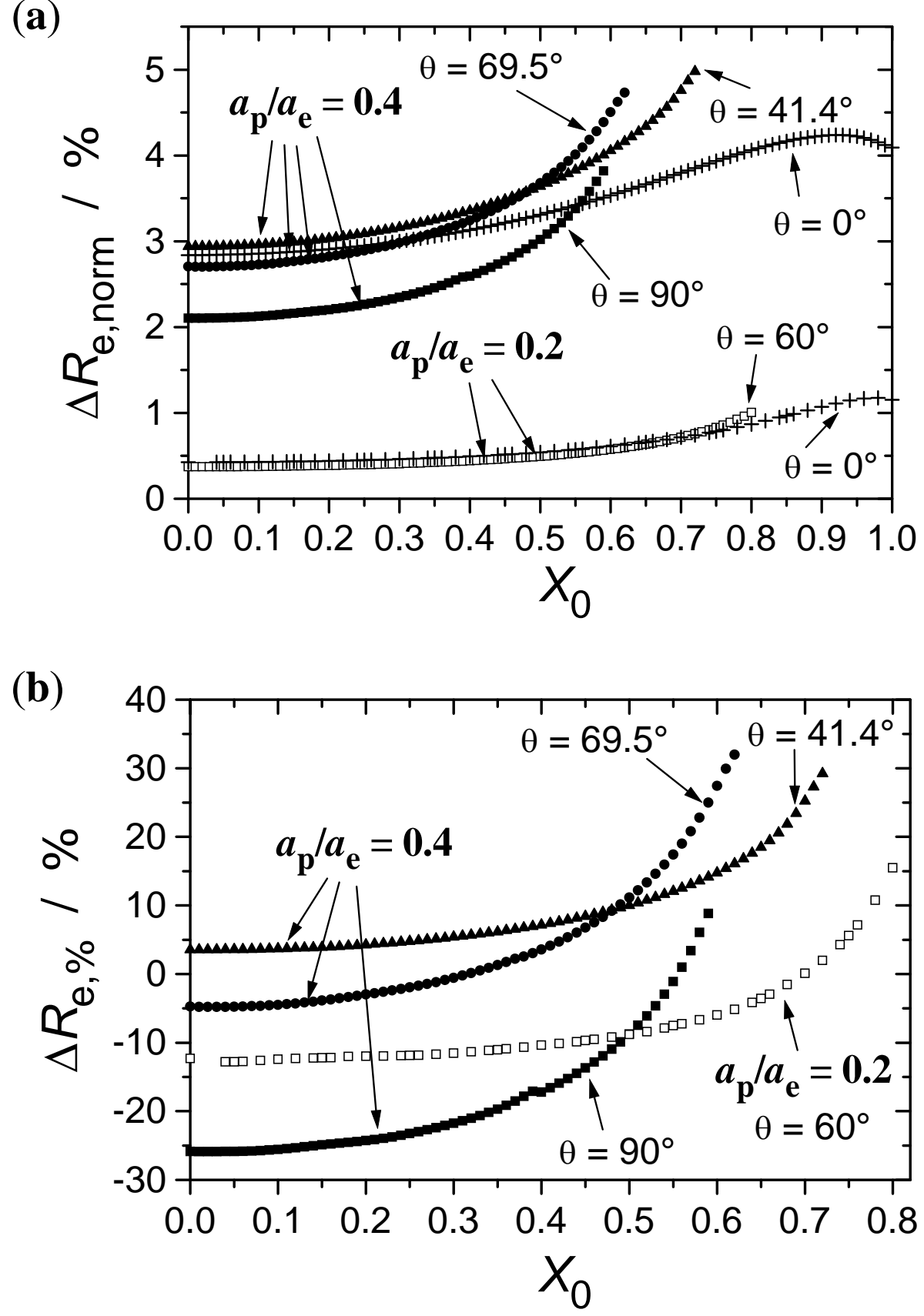

Fig. 4. Normalized ER increment $\Delta R_{\mathrm{e}, \text { norm }}$ (a) and $\Delta R_{\mathrm{e}, \%}$ (b) due to the presence of an insulating sphere of various contact angle and size $\left(a_{\mathrm{p}} / a_{\mathrm{e}}=0.2\right.$ and 0.4$)$ as a function of its radial position $X_{0}$. 


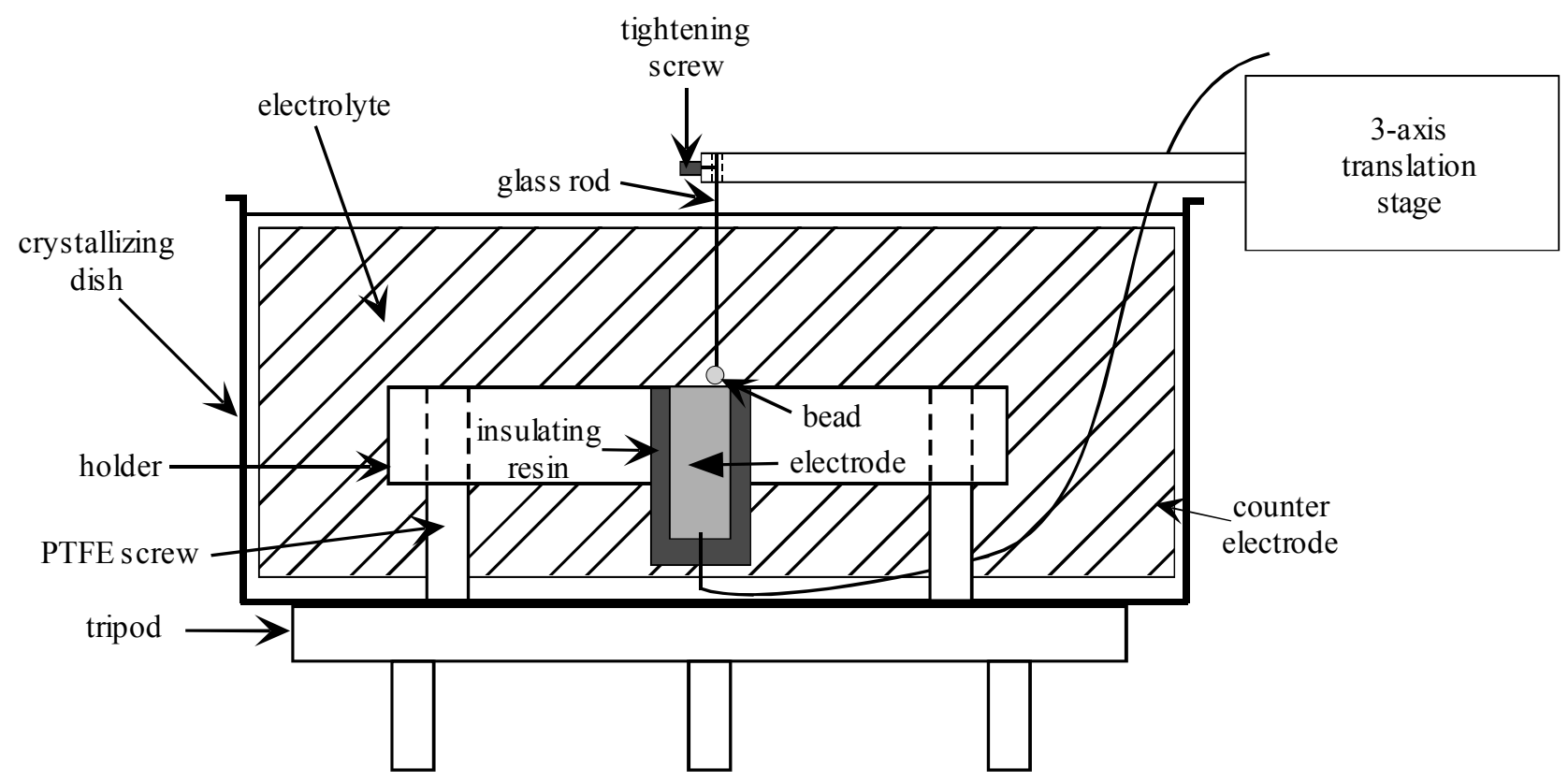

Fig. 5. Experimental set-up used for measuring the ER variations due to the presence of a sphere shifted above or in contact with the electrode. 

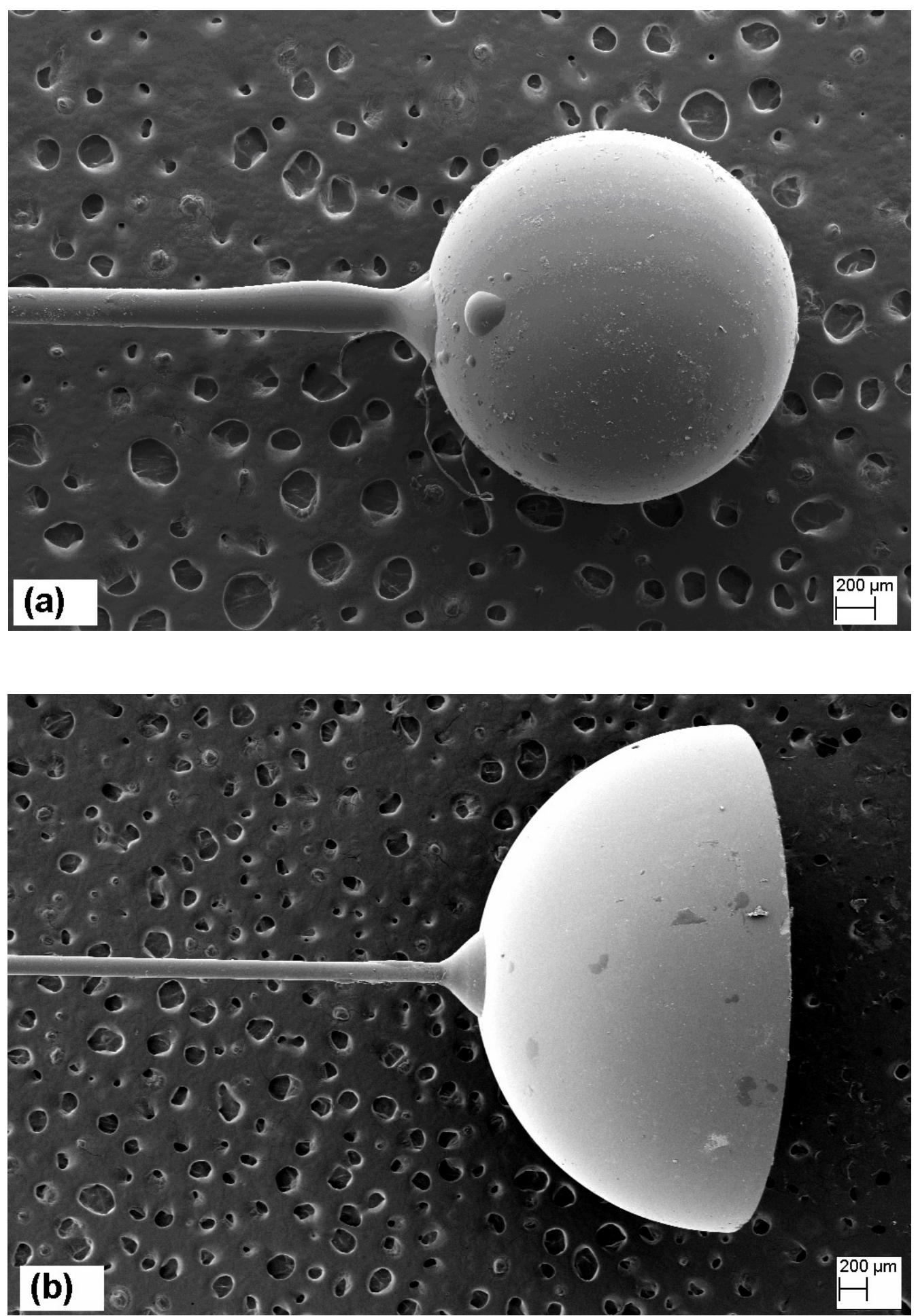

Fig. 6. SEM images of the sphere of diameter $2 \mathrm{~mm}$ (a) and hemisphere $\left(\theta=90^{\circ}\right)$ of diameter 4 $\mathrm{mm}(\mathrm{b})$. 

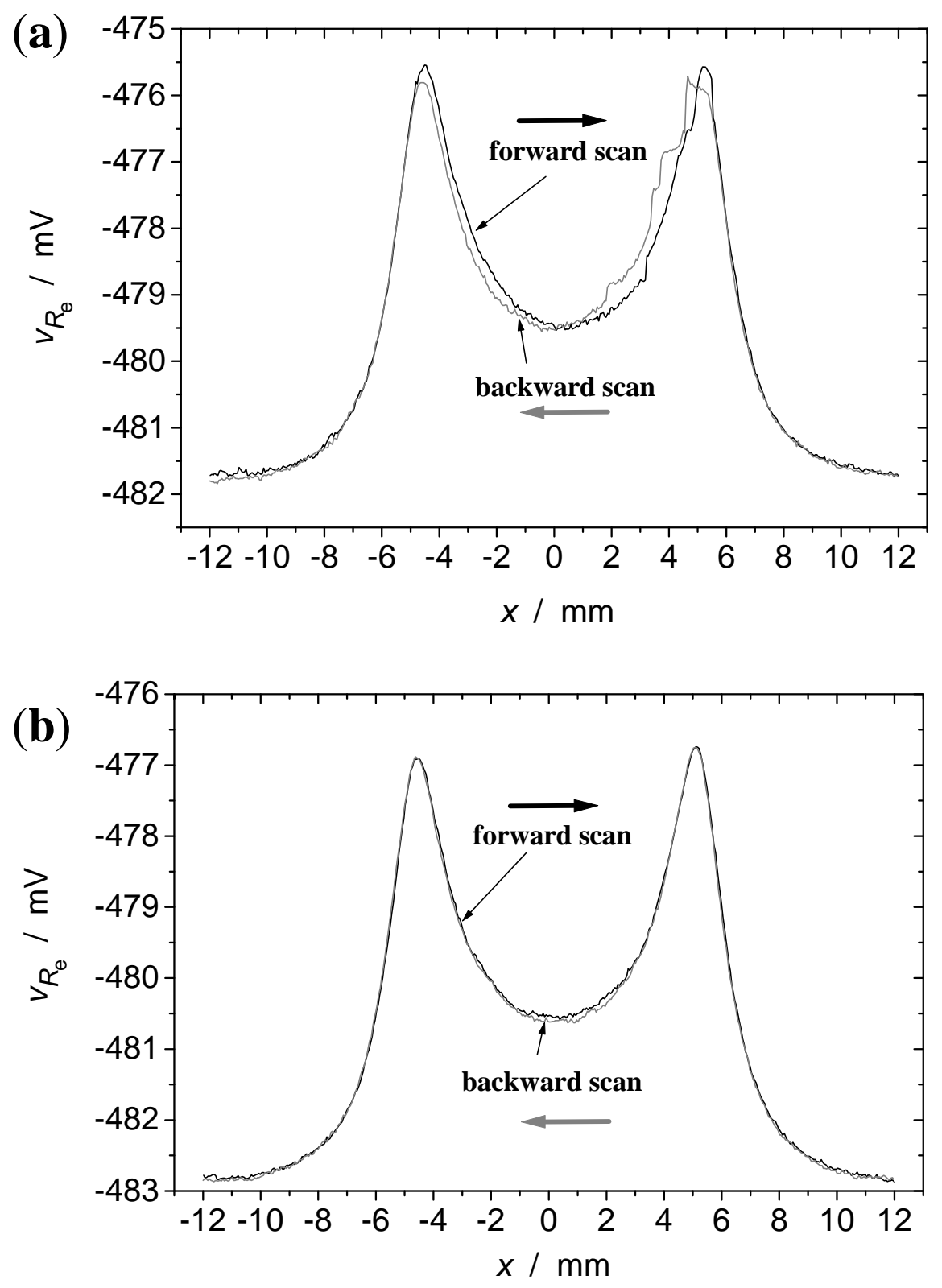

Fig. 7. Variation of $v_{R_{\mathrm{e}}}$ as a function of the radial position $x$ of an insulating 2-mm-diameter sphere plane (a) in contact with $(h=0)$ and $(\mathrm{b})$ above $(h=3 \mu \mathrm{m})$ a 10 -mm-diameter stainless steel electrode during a forward and backward scan at a $0.4 \mathrm{~mm} / \mathrm{s}$ rate. 


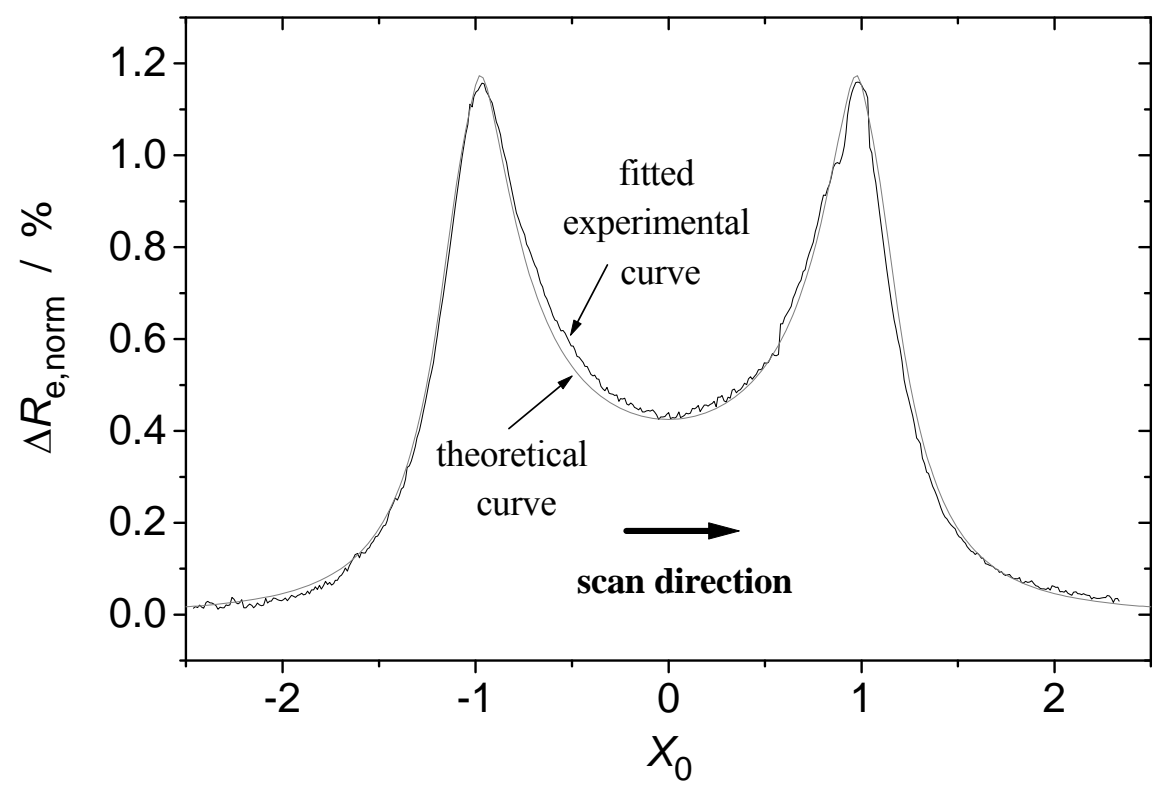

Fig. 8. Theoretical and experimental normalized $\Delta R_{\mathrm{e}, \text { norm }}$ variations as a function of the normalized radial position $X_{0}$ of an insulating 2-mm-diameter sphere in contact with the electrode. The experimental data correspond to the forward scan in Fig. 7a. 
(a)
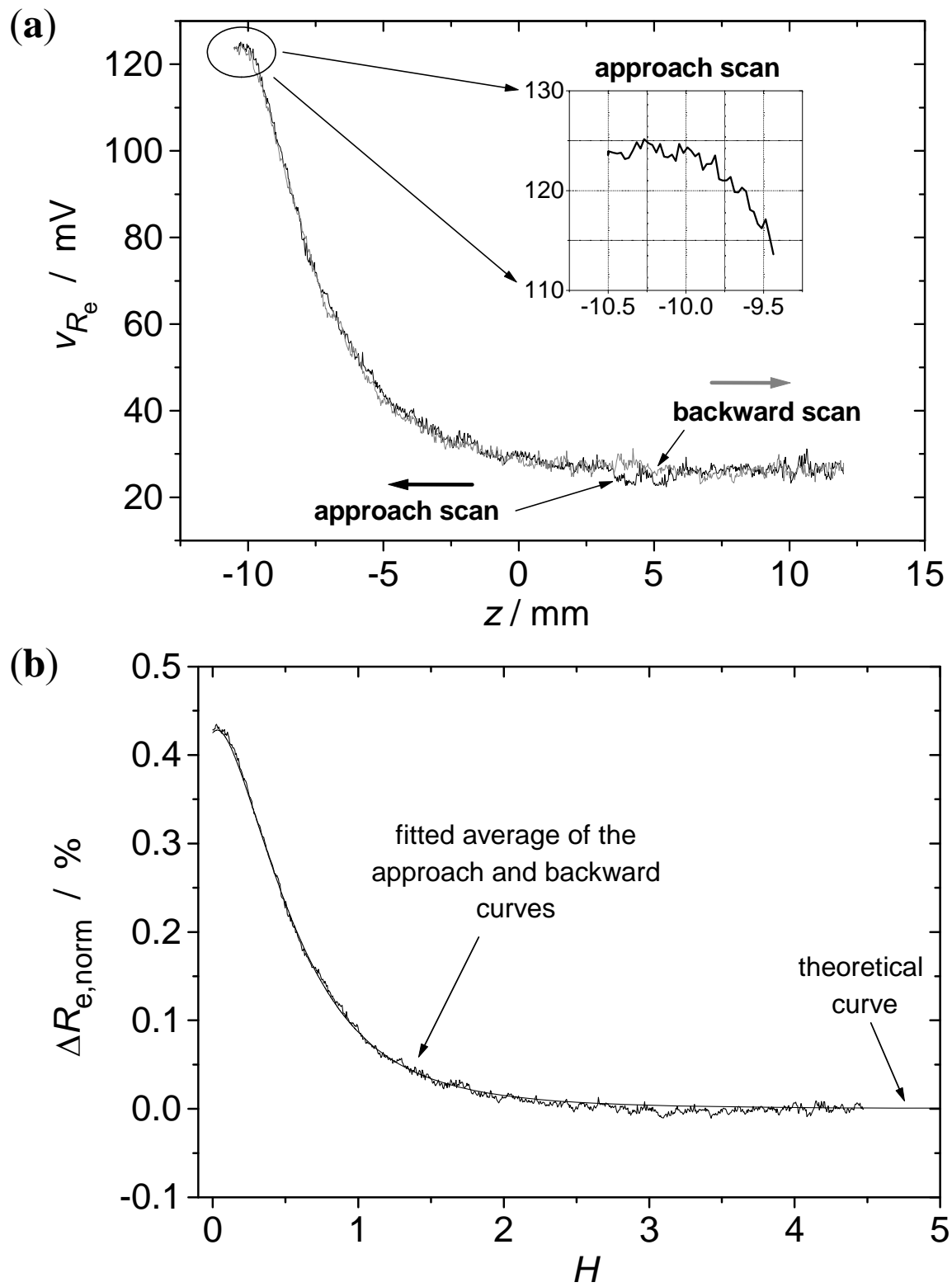

Fig. 9. (a) Variation of $v_{R_{\mathrm{e}}}$ as a function of the vertical position $z$ of an insulating 2-mm-diameter sphere above the electrode centre for an approach and backward scan at a $0.2 \mathrm{~mm} / \mathrm{s}$ rate; (b) experimental normalized $\Delta R_{\text {e,norm }}$ variations (average of the approach and backward curves) fitted with the theoretical curve, as a function of the normalized vertical position $H$ of the sphere above the electrode. 

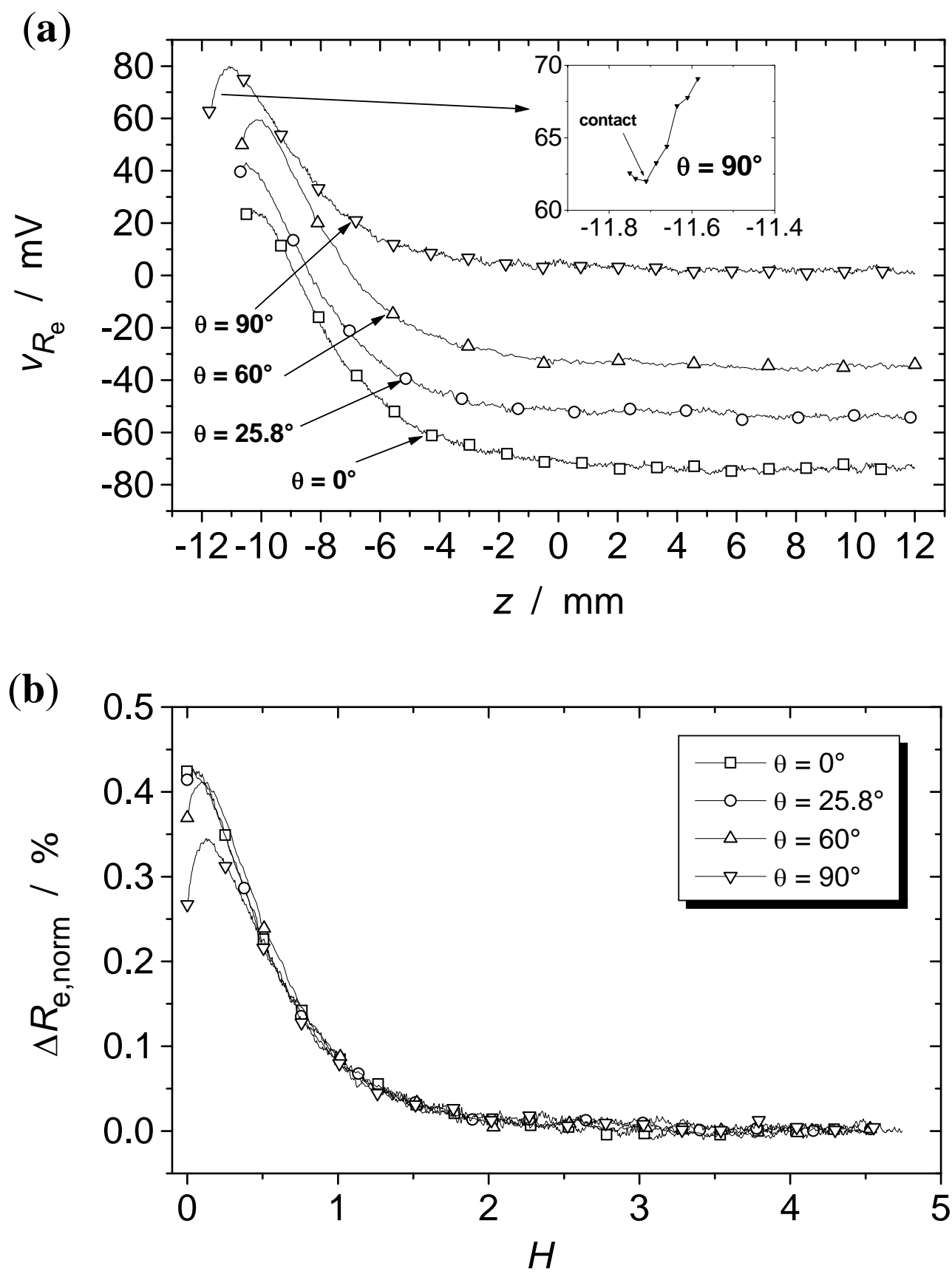

Fig. 10. (a) Variation of $v_{R_{\mathrm{e}}}$ as a function of the vertical position $z$ and (b) normalized ER increment $\Delta R_{\mathrm{e}, \text { norm }}$ as a function of the normalized height $H$ of insulating 2-mm-diameter spheres with different contact angles above the electrode centre $(0.2 \mathrm{~mm} / \mathrm{s} \mathrm{scan}$ rate $)$. 

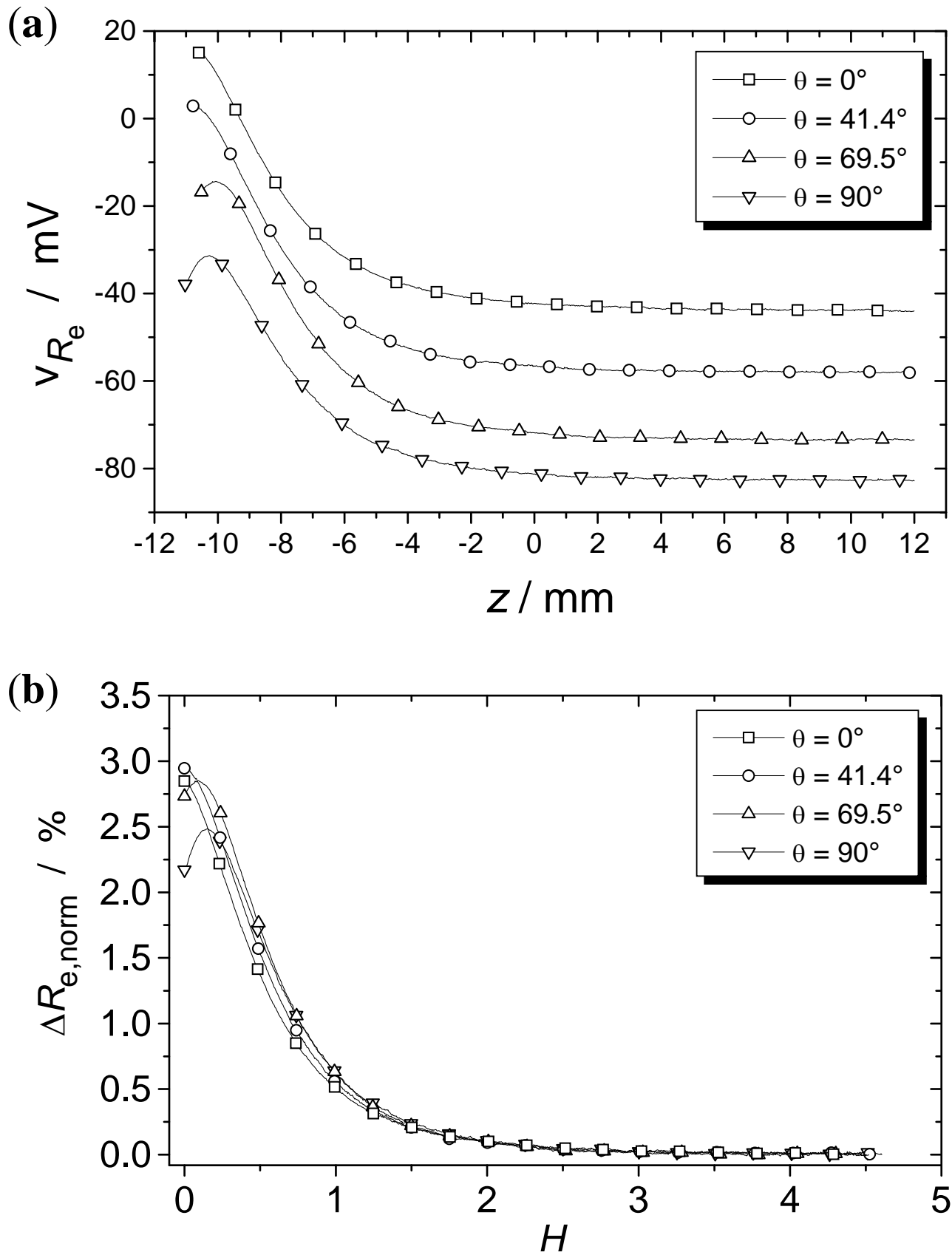

Fig. 11. (a) Variation of $v_{R_{\mathrm{e}}}$ as a function of the vertical position $z$ and (b) normalized ER increment $\Delta R_{\mathrm{e}, \text { norm }}$ as a function of the normalized height $H$ of insulating 4-mm-diameter spheres with different contact angles above the electrode centre $(0.2 \mathrm{~mm} / \mathrm{s} \mathrm{scan}$ rate $)$. 


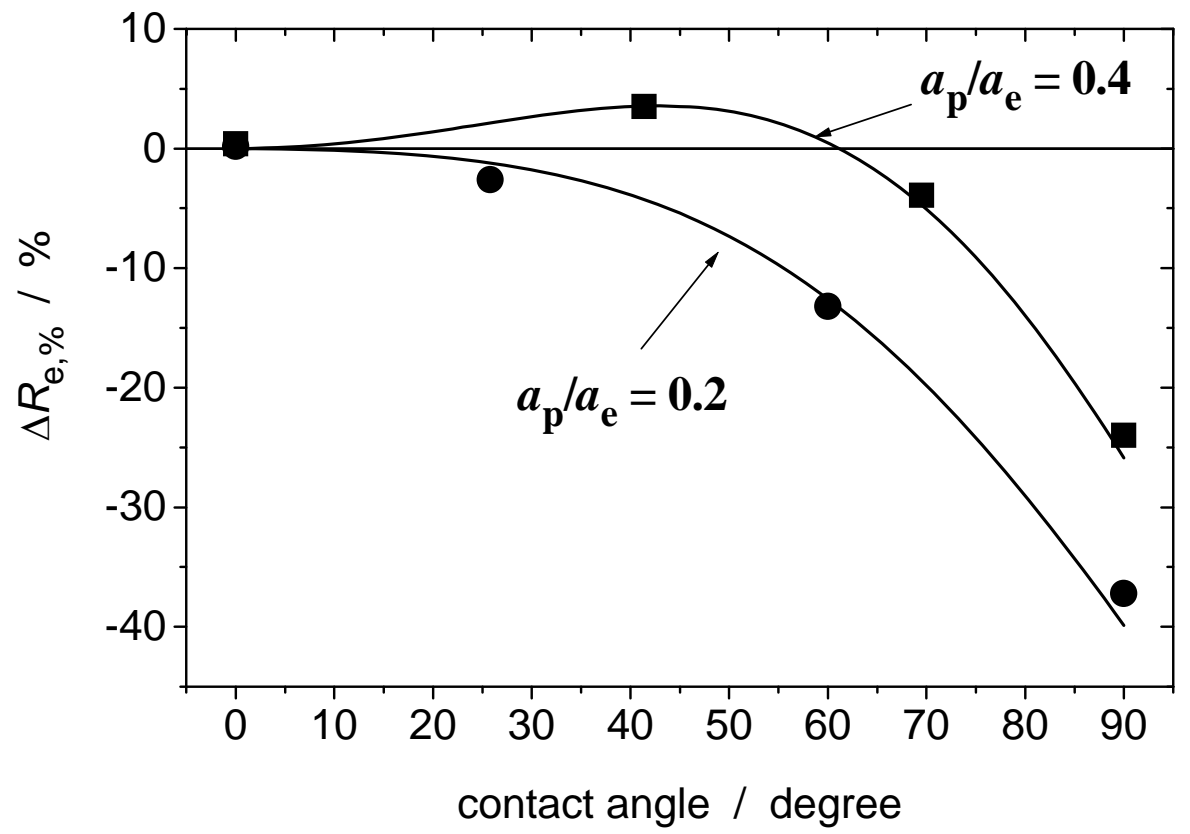

Fig. 12. Theoretical and experimental normalized ER increment $\Delta R_{\mathrm{e}, \%}$ as a function of the contact angle of the spheres of $2 \mathrm{~mm}$ and $4 \mathrm{~mm}$ in diameter in contact with the electrode centre. 